\title{
Morbidity rates of complications in persons with spinal cord injury according to the site of injury and with special reference to hypertension
}

\author{
K Imai PHN,${ }^{1} \mathrm{~T}$ Kadowaki $\mathrm{PhD},{ }^{1} \mathrm{Y}$ Aizawa $\mathrm{MD},{ }^{2} \mathrm{~K}$ Fukutomi $\mathrm{PhD}^{3}$ \\ ${ }^{1}$ Department of Public Health, School of Nursing, ${ }^{2}$ Department of Preventive Medicine \\ and Public Health, School of Medicine, Kitasato University, 2-1-1 Kitasato, Sagamihara, \\ Kanagawa, 228 Japan; ${ }^{3}$ The Institute of Public Health, Shirogane, Minato-ku, Tokyo, \\ Japan.
}

It has been reported that since 1982 the incidence in persons with spinal cord injury (SCI) of hypertension is commoner than it is in the general population of the same age groups in Japan. In the current study, we examined outpatient morbidity rates and standardised outpatient morbidity ratios (SOMR) according to the site of injury, as well as blood pressure levels and history of disease, and compared the incidences with those for the general population. The subjects consisted of 195 men with SCI. All were engaged in light work at special centres while living with other persons at the centres. The mean age was 49.5 years old, and the average post-injury period was 17.9 years. With respect to the site of injury, 19 patients had had injuries at the level of C-T5, 24 at T6-T10, 139 at T11-L1, and 13 at L2 or lower. The SOMR (general population $=100$ ) for hypertension was closely related to the site of injury, i.e. 0 at C-T5, 250 at T6-T10, 221 at T11-L1 and 308 at L2 or below. Among the patients treated with antihypertensive agents (41 persons), $17.1 \%$ were under treatment for renal diseases, $4.9 \%$ for diabetes, and $4.9 \%$ for hepatic disorders. In $68 \%$ of the SCI persons examined, however no disease (such as renal disease, diabetes mellitus, hepatic disease or endocrine abnormality) could be regarded as a cause of secondary hypertension. In addition, the survey revealed that the body weight of SCI persons was lower than that of the general populations.

Keywords: spinal cord injury; morbidity; prevalence; hypertension; diabetes mellitus.

\section{Introduction}

Advanced treatment for both acute and chronic spinal cord injuries has rapidly decreased the morbidity rate of complications in the urinary system, but the morbidity and mortality rates of circulatory diseases have increased. ${ }^{1-5}$ It has been reported that, of circulatory diseases in persons with SCI since 1982, hypertension was more frequently encountered when compared to the general population of the same age groups in Japan. ${ }^{6-9}$

In order to compare the morbidity rate for SCI outpatients with that for the general population, a survey in accordance with the National Livelihood Basic Survey (1989) ${ }^{10}$ that is performed at 3 year intervals by the Ministry of Health and Welfare was carried out on SCI persons. The SCI persons had completed medical rehabilitation and were engaged in light work at special centres. For comparison, the standardised outpatient morbidity ratios (SOMR), an age-adjusted indicator based on the values for the general population, was obtained according to the site of injury.

\section{Study methods}

\section{Survey principles}

Physical examinations and questionnaires were based on the section related to health in the 1989 National Livelihood Basic Survey (Ministry of Health and Welfare). ${ }^{10}$

The same questionnaire contents as those in the health section of the 1989 survey were 
used. The surveyed items included diseases being treated at hospitals or clinics at the time of the survey, and the data of general health status for the general population was used as the control. The survey of SCI persons was conducted in accordance with the principle of the 1989 survey.

A survey of blood pressure, body height, body weight, disease history and administration of antihypertensive drugs was entrusted to eight special centres in 1990 . For controls, data for the general population from the National Nutrition Survey ${ }^{11}$ were used.

\section{Subjects}

195 men with SCI who satisfied both of the following conditions were surveyed: (1) those who gave effective answers to the health questionnaire that was distributed to 307 subjects ( 244 replies were received), and (2) those who had undergone blood pressure determinations and an investigation of their medical history (228 persons).

All the subjects were engaged in light work at the special centres while living with other persons at the centres.

The SCI persons were 49.5 years old on average, the average post-injury period being 17.9 years. With respect to the site of injury, 19 patients had had injuries at the level of C-T5, 24 at T6-T10, 139 at T11-L1, and 13 at L2 or lower.

\section{Calculation method}

The following indicators were calculated with respect to 'diseases being treated at outpatient clinics' in the health questionnaire, and compared according to the site of injury: (1) outpatient rate $=$ (number of outpatients/population studied) $\times 1000$ and (2) standardised outpatient morbidity ratio.

The disease-specific ratio of SCI outpatients to the outpatient of the general population was used as the standard (100), namely, an age-adjusted indicator calculated by the indirect method as a standardised outpatient morbidity ratio (SOMR), which was calculated according to the following equation:

SOMR $=$ (total number of outpatients/ expected number of outpatients) $\times 100$
The expected number of outpatients can be calculated from the following equation:

Expected number of outpatients $=\sum_{(\mathrm{i})}$

(the number of persons in age group $\mathrm{i}$ of the population studied) $\times$ (the outpatient rate in age group $i$ of the general population), where $\sum_{(\mathrm{i})}$ denotes summation over all age groups.

The results from the 1989 National Livelihood Basic Survey were used to provide SOMR.

The methods used for testing the significance of SOMR were as follows:

(1) For 10 or more SCI outpatients

$$
\begin{array}{r}
([\text { observed value }- \text { expected value }]-0.5) / \\
\sqrt{\text { expected value }}
\end{array}
$$

This was tested by using a normal distribution.

(2) For less than 10 SCI outpatients, a probability was calculated on the basis of the Poisson distribution.

\section{Results}

Outpatient rate and SOMR according to the site of injury

Since the incidence of outpatients with cystitis was higher in SCI persons, and extremely low in the general population, the SOMR vs the general population (100) was as high as 6901 to 15674 (Table I).

Although the numbers of persons with injuries at C-T5 and T6-T10 were small, the SOMR was significantly higher with respect to complications in the urinary tract and to gastrointestinal diseases $(p<0.01)$.

Since there was a large number of persons with thoracolumbar injuries (T11-L2), the SOMR was significant for many diseases. This ratio was significant for urinary tract diseases, skin diseases, hypertension and diabetes $(p<0.01)$, and also significant for injuries involving burns, prostatic hypertrophy and hypotension $(p<0.05)$.

The outpatient rate in persons with injuries at L2 or lower (24 persons) was the highest for hypertension, the SOMR was significant for renal diseases, neuralgia, 
Table I Outpatient rates and standardised outpatient morbidity ratios for SCI persons according to the level of the lesion

\begin{tabular}{lrrr}
\hline & No. & Prev. & SOMR \\
\hline C-T5 (19 persons) & & & \\
Cystitis & 5 & 263 & $24913^{\mathrm{b}}$ \\
Other diseases of the & 3 & 159 & $2406^{\mathrm{b}}$ \\
$\quad$ digestive system & & 105 & $2049^{\mathrm{b}}$ \\
Renal diseases & 2 & & \\
T6-T10 (13 persons) & & & \\
Cystitis & 6 & 250 & $19352^{\mathrm{b}}$ \\
Renal diseases & 6 & 250 & $4187^{\mathrm{b}}$ \\
Hypertension & 5 & 208 & 300 \\
Gastric ulcer and & 4 & 167 & $1194^{\mathrm{b}}$ \\
$\quad$ duodenal ulcer & & & \\
Other diseases of the & 2 & 83 & $3024^{\mathrm{b}}$ \\
$\quad$ urinary system & & & \\
Other diseases of the & 2 & 83 & $1124^{\mathrm{a}}$ \\
$\quad$ digestive system & & & \\
T11-L2 (139 persons) & & & \\
Cystitis & 27 & 194 & $15674^{\mathrm{b}}$ \\
Hypertension & 21 & 151 & $221^{\mathrm{b}}$ \\
Renal diseases & 18 & 129 & $2194^{\mathrm{b}}$ \\
Disorders of the skin & 10 & 72 & $450^{\mathrm{b}}$ \\
Other diseases of the & 9 & 65 & $2463^{\mathrm{b}}$ \\
$\quad$ urinary system & & & \\
Diabetes mellitus & 9 & 65 & $326^{\mathrm{b}}$ \\
Injuries and burns & 4 & 29 & $530^{\mathrm{a}}$ \\
Hypertrophy of the prostate & 3 & 22 & $610^{\mathrm{a}}$ \\
Hypotension & 3 & 22 & $607^{\mathrm{a}}$ \\
$\begin{array}{l}\text { L2- (24 persons) } \\
\text { Hypertension }\end{array}$ & & & \\
Renal diseases & 5 & 385 & $697^{\mathrm{b}}$ \\
Neuralgia & 4 & 308 & $5569^{\mathrm{b}}$ \\
$\begin{array}{l}\text { Diabetes mellitus } \\
\text { Cystitis }\end{array}$ & 2 & 154 & $1947^{\mathrm{b}}$ \\
\hline No. & 1 & 77 & $695^{\mathrm{b}}$ \\
\hline & & $691^{\mathrm{a}}$ \\
\hline
\end{tabular}

No. = number of outpatients; Prev. = outpatient rate (per 1000); SOMR = standardised outpatient morbidity ratio (general population $=100)$.

Statistical significant at ${ }^{\mathrm{a}} p<0.05$ and ${ }^{\mathrm{b}} p<0.01$ compared with the general population.

hypertension and diabetes $(p<0.01)$, and for cystitis $(p<0.05)$.

Blood pressure according to the age and site of injury

The mean diastolic blood pressure levels in SCI persons were $76.5,79.4$ and $77.6 \mathrm{mmHg}$ for people in their $40 \mathrm{~s}, 50 \mathrm{~s}$ and $60 \mathrm{~s}$ respect- ively, compared with a value of around $83 \mathrm{mmHg}$ for the general population between 40 and 69 years of age, demonstrating lower values for SCI persons in all age groups. The mean systolic blood pressure was also lower in SCI persons $(123.3,134.2$, and $135.9 \mathrm{mmHg}$ for people in their $40 \mathrm{~s}, 50 \mathrm{~s}$ and 60 s respectively, compared with 131.5 , 137.3 and $142.9 \mathrm{mmHg}$ for the general population in the same age group).

With respect to the average blood pressure according to the site of injury, both the diastolic and systolic blood pressure levels were higher in those persons with injuries at L2 or lower (Fig 1).

\section{Body weight and degree of obesity}

The mean body weight of SCI persons aged over 40 years was around $55 \mathrm{~kg}$, being lower than the $64 \mathrm{~kg}, 62 \mathrm{~kg}$ and $60 \mathrm{~kg}$ averages for the general population in their $40 \mathrm{~s}, 50 \mathrm{~s}$, and 60 s respectively. The degree of obesity in SCI persons (Katsura's modification of the Broca method) was also relatively low at $6.8 \%, 2.5 \%$ and $1.0 \%$ for their $40 \mathrm{~s}, 50 \mathrm{~s}$, and 60 s respectively.

Although the body weight of SCI persons treated with antihypertensive agents was

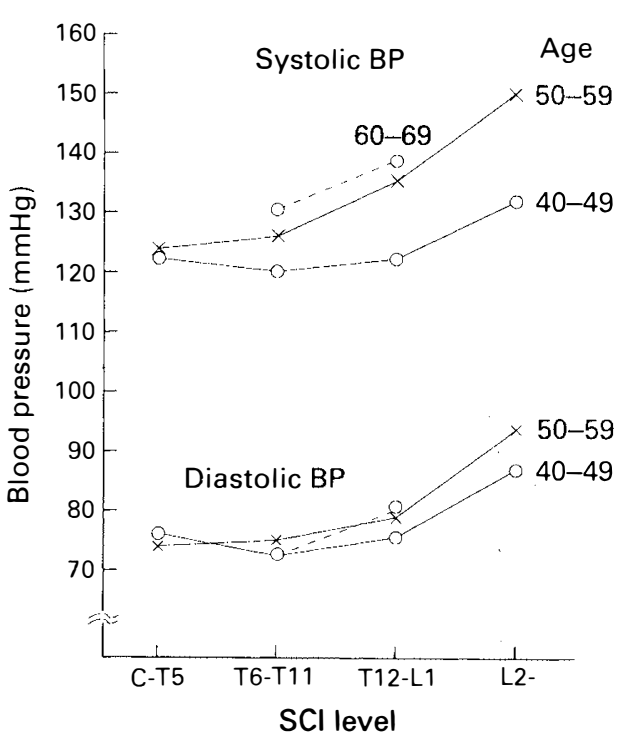

Figure 1 Blood pressure of SCI patients according to the level of the lesion and age group. 
slightly higher than that of untreated persons in the same age group (the difference was significant at $0.01 \%$ in those in their 40 s, but not significant for those in their 50s and $60 \mathrm{~s}$ ), but lower than the figures for the general population in the same age groups.

\section{Percentage of patients treated with antihypertensive agents}

The percentage of SCI persons treated with antihypertensive agents was compared with the percentage of those treated with these agents in the general population. A total of 41 SCI persons had been treated with antihypertensive agents, the percentage being $9.1 \%$ (compared with $2.0 \%$ of the general population), of which $15.6 \%$ $(5.1 \%), 21.8 \%(14.4 \%)$ and $45.5 \%(22.8 \%)$ were in their $30 \mathrm{~s}, 40 \mathrm{~s}, 50 \mathrm{~s}$ and $60 \mathrm{~s}$ respectively.

Figure 2 shows the percentages of patients treated with antihypertensive agents according to the site of injury and age. The percentage of persons treated with the agents was higher among SCI persons than for the general population, except for those patients with injuries at C-T5. The percentage was especially high among persons with

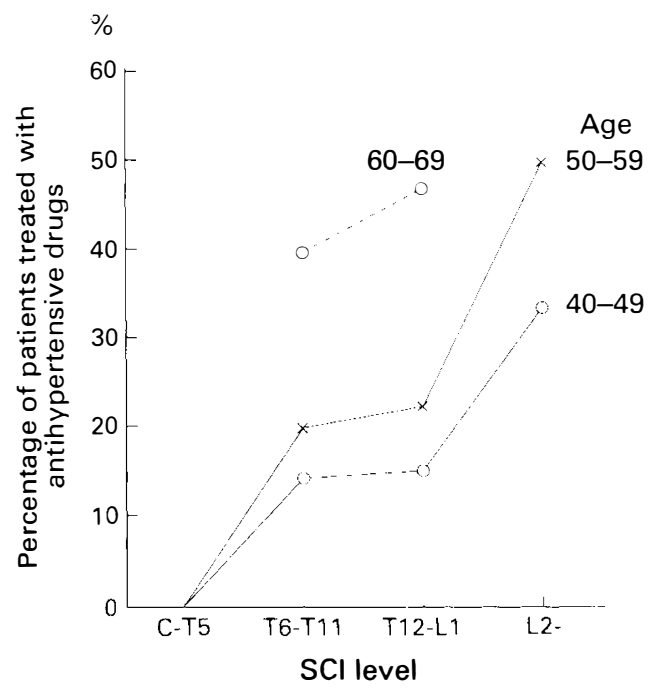

Figure 2 Percentage of SCI patients with hypertension who have been treated with antihypertensive drugs. injuries at L2 or lower $(33.3 \%$ and $66.7 \%$ for their 40s and 50s respectively).

\section{Frequency of complications from}

hypertension and other diseases

Among the 41 patients treated with antihypertensive agents, seven $(17.1 \%)$ were under treatment for renal disorders. Moreover, two patients $(4.9 \%)$ were under treatment for diabetes, while three $(7.3 \%)$, although not being treated, had a past history of diabetes. Two patients $(4.9 \%)$ were having treatment for hepatic disorders.

\section{Discussion}

A factor most closely related to hypertension in the general population is advanced age. The percentage of SCI persons having antihypertensive treatment also increases with advancing age. ${ }^{10}$ For a more precise comparison by excluding this agerelated influence, the SOMR was obtained. The overall SOMR for hypertension was 250 , although that was closely related to the site of injury, i.e. 0 at C-T5, 250 at T6-T10, 221 at T11-L1 and 308 at L2 or below.

In SCI persons injured at a high level, orthostatic hypotension and autonomic hyperreflexia ${ }^{12-13}$ are often cited as complications. Hypertensive attack due to autonomic hyperreflexia is transient in nature and chronically shows a trend of hypotension due to the decreased tonus of sympathetic nerves. For this reason, SCI persons injured at $\mathrm{T} 5$ or above would have shown low SOMR for hypertension.

Urinary diseases, being the most important secondary complication, are apt to occur when the initial treatment is inadequate or when the urinary management in daily life is not adequately performed. ${ }^{14-16}$ In the results of this survey, the incidence of complicated renal diseases including pyelonephlitis, nephritis, hydronephrosis and renal failure was $17 \%$. However, many patients had repeated urinary infection without self recognition, suggesting the possibility of a latent renal disturbance that could not be detected by a routine examination. 
Although abnormal lipids in SCI patients with chronic renal failure (CRF) have been reported elsewhere, ${ }^{17}$ it is still unclear how such latent CRF participates in the onset of hypertension.

Apart from renal disease, diabetes mellitus and hepatic disorders are also cited as causes of secondary hypertension. In connection with diabetes mellitus, Duckworth et $a l^{18,19}$ and Bauman et $a l^{20}$ have reported an abnormal glucose tolerance capacity in SCI persons, which was considered to be due in part to insulin resistance. In the present survey, the difference in SOMR for diabetes mellitus, according to the site of injury, was also marked. Therefore, diabetes mellitus was also closely related to the site of injury.

The incidence of complicated hepatic disorders in this survey was significantly high in SCI persons as a whole, being approximately 3.7 times higher than the figure for the general population. ${ }^{9}$ This was also found by Nakajima et $a l^{6}$ and may be partially attributed to the high morbidity rate of post-transfusion hepatitis during the course of treatment for SCI.

In the present survey, in $68 \%$ of the SCI persons examined, however, no diseases that can be regarded as a cause of secondary hypertension such as renal disease, diabetes mellitus, hepatic disease or endocrine abnormality were observed.

In addition to the complications already mentioned, obesity and lipid abnormality, as well as arteriosclerosis caused by these conditions, are listed as a factor probably involved in the onset of hypertension. The survey revealed that the body weight of SCI persons was lower than that of the general populations with a higher incidence of hypertension irrespective of the lower rate of obesity found in the former group. The influences of a widely extended mass of muscle for SCI persons have not been clarified. Consequently, it is difficult to compare directly the results for SCI persons with the figures for the general population, even if the degree of obesity is low. ${ }^{21,22}$ For a more accurate comparison, it is necessary to assess the degree of obesity according to the amount of muscle in the whole body and the rate of somatic lipids.
High morbidity rates for heart diseases in SCI persons have been reported by some investigators ${ }^{4,23,24}$ Regarding lipid metabolism, lipid abnormality and especially low HDL-cholesterol in SCI persons are cited. ${ }^{20,23,24}$ However, Cardus et $a l^{25}$ has reported no difference, and the possible involvement of lack of exercise and the paralysis caused by SCI may have to be taken into consideration concerning this point. ${ }^{26}$

No significant difference was noted in the SOMR for heart diseases in this survey, although the value is expected to increase in the future for the general population because of the changing diet in Japan in recent years to include high protein and high animal lipids. ${ }^{11}$

Regarding somatic activities, all SCI persons enrolled in this survey were confined to a life in a wheelchair and were engaged in light, simple work to obtain some income. Due to this handicap, while they have many opportunities to use their upper limbs, they have little chance to exercise the whole body. A higher HDL-cholesterol level in SCI persons taking exercise when compared to those not taking exercise has been reported,${ }^{27}$ and it is thus also considered that a lack of exercise would directly affect the level of blood pressure in SCI persons.

The amount of ingested salts is important as a dietary factor pertaining to the onset of hypertension. The subjects enrolled in this survey all lived in special centres, and their diets were managed by a professional nutritionist to give a lower salt intake than that for the general population. For this reason, little influence from excessive salt intake is suspected.

Another factor triggering hypertension in SCI persons is the influence of stress due to social psychological factors. In our previous survey on subjective symptoms, neurological symptoms including pain and insomnia, and mental symptoms including nervousness and depression were characteristically more often encounted in SCI patients than in the general population (accurate judgement rates for control were $85 \%$ and $75 \%$ respectively). ${ }^{28}$ Although a possible relationship between a depressive state and endocrine abnormality has been 
reported, ${ }^{29}$ a prolonged state of mental instability may also affect the blood pressure level in SCI persons.

The fact that the SOMR for hypertension markedly increased with the descending site of injury indicates that primary and/or secondary disturbances after SCI may be linked with the occurrence of hypertension in SCI persons, in addition to the daily life factors already mentioned.

\section{References}

1 DeVivo MJ, Stover SL, Black KJ (1992) Prognostic factors for 12-year survival after spinal cord injury. Arch Phys Med Rehabil 73: 156-162.

2 Whiteneck GG, Charlifue SW, Frankel HL et al (1992) Mortality, morbidity, and psychosocial outcomes of persons spinal cord injured more than 20 years age. Paraplegia 30: 617-630.

3 Lang HD, Durr W, Hoffman J, Koeth R (1980) Post-clinical follow-up of spinal patients through domestic check-ups. Paraplegia 18: 140-141.

4 Yekutiel M, Brooks ME, Ohry A, Yarom J, Carel R (1989) The prevalence of hypertension, ischemic heart disease and diabetes in traumatic spinal cord injured patients and amputees, Paraplegia 27: 58-62.

5 Sakai M, Yoshida I, Itoh F et al (1988) Aging in persons with spinal cord injuries. JJTOM 31: 659-663 (in Japanese).

6 Nakajima A, Honda S, Yoshimura S et al (1989) The disease pattern and causes of death of spinal cord injured patients in Japan. Paraplegia 27: 163-171.

7 Kadowaki T (1982) Status of health in persons with traumatic spinal cord lesion. JJTOM 30: 805-815 (in Japanese).

8 Nakajima A, Yoshida I, Itoh F et al (1982) Aging in persons with spinal cord injuries. JJTOM 30: 536-540 (in Japanese).

9 Imai K, Kadowaki T, Takata T, Fukutomi K. (1993) Comparison of diseases prevalence between spinal cord injury persons and the general population. J Jpn Med Soc Paraplegia 5: 140-141 (in Japanese).

10 Statistics and Information Department, Minister's Secretariat, Ministry of Health and Welfare (1989) National Livelihood Basic Survey. Tokyo.

11 Health Service Bureau, Ministry of Health and Welfare (1993) 1991 National Nutrition Survey. Tokyo.

12 Braddom RL, Rocco JF (1991) Autonomic dysreflexia. A survey of current treatment. Am J Phys Med Rehabil 70: 234-241.

13 Lindan R, Joiner E, Freehafer AA, Hazel C (1980) Autonomic dysreflexia in patients with spinal cord injury. Paraplegia, 18; 285-292.

14 Killorin W, Gray M, Bennett JK, Green BG (1992) The value of urodynamics and bladder management in predicting upper urinary tract complications in male spinal cord injury patients. Paraplegia 30: 437-441.

15 Stover SL, Lloyd LK, Waites KB, Jackson AB (1991) Neurogenic urinary tract infection. Neurogen Clin 9: 741-755.

16 Hoffberg HJ, Cardenas DD (1986) Bladder trabeculation in spinal cord injury. Arch Phys Med Rehabil 67: 750-753.

17 Vaziri ND, Gordon S, Nikakhtar B (1982) Lipid abnormalities in chronic renal failure associated with spinal cord injury. Paraplegia 20: 183-189.

18 Duckworth WC, Jallepalli P, Solomon SS (1983) Glucose intolerance in spinal cord injury, Arch Phys Med Rehabil 64: 107-110.

19 Duckworth WC, Solomon S, Jallepalli P et al (1980) Glucose intolerance due to insulin resistance in patients with spinal cord injuries. Diabetes 29: 906-910.

20 Bauman WA, Spungen AM, Raza M et al (1992) Coronary artery disease: Metabolic risk factors and latent disease in individuals with paraplegia. Mt Sinai J Med 59: 163-168.

21 Kuhlemeier KV, McEachran AB, Lloyd LK (1984) Serum creatinine as an indicator of renal function after spinal cord injury. Arch Phys Med Rehabil 65: 694-697.

22 Mirahmadi MK, Byrne C, Barton C (1983) Prediction of creatinine clearance from serum creatinine in spinal cord injury patients. Paraplegia 21: 23-29.

23 Krum H, Howes LG, Brown DJ et al (1992) Risk factors for cardiovascular disease in chronic spinal cord injury patients. Paraplegia 30: 381-388.

24 Bauman WA, Spungen AM, Zhong YG et al (1992) Depressed serum high density lipoprotein cholesterol levels in veterans with spinal cord injury. Paraplegia 30: 697-703.

25 Cardus D, Ribas-Cardus F, McTaggart WG (1992) Lipid profiles in spinal cord injury. Paraplegia 30: $775-782$.

26 Cardis D, Ribas-Cardús F, McTagget WG (1992) Coronary risk in spinal cord injury: Assessment following a multivariate approach. Arch Phys Med Rehabil 73: 930-933.

27 Brenes G, Dearwater S, Shapera R (1986) High density lipoprotein cholesterol concentrations in physically active and sedentary spinal cord injured patients. Arch Phys Med Rehabil 67: 445-450. 
28 Kadowaki T, Imai K, Fukutomi K et al (1991) Cornal Medical Index analysis of subjective symptoms in persons with traumatic spinal cord injury. JJTOM 39: 272-279.

29 Palmer JB (1985) Depression and adrenocortical function in spinal cord injury patients. Arch Phys Med Rehabil 66: 253-256. 